\title{
The potential hazard of a non-slip element balloon causing distal longitudinal stent deformation: The first clinical experience and in vitro assessment
}

\author{
Hiroki Shibutani, Yuzo Akita, Yohei Oishi, Hiroyuki Sueyoshi, Yu Mukai, \\ Kotaro Yutaka, Yumie Matsui, Masahiro Yoshinaga, Masahiro Karakawa \\ Division of Cardiology, Osaka Saiseikai Izuo Hospital, Osaka, Japan
}

\begin{abstract}
Background: A new complication, longitudinal stent deformation (LSD), is increasingly reported with recent intracoronary stent designs. There have been experiences of unusual cases of distal LSD caused by entrapment of a Lacrosse ${ }^{\circledR}$ non-slip element (NSE) balloon (Goodman Co., Ltd., Nagoya, Japan), which has three flexible nylon elements to prevent slippage. Accordingly, the aim of this study is to report the clinical experience of distal LSD caused by the NSE in the documented center and to investigate the incidence and mechanisms involved.

Methods: Coronary intervention cases were retrospectively reviewed using the NSE balloon in hospital between May 2014 and June 2017. In bench testing, distal LSD was reproduced in a silicon tube model to identify its mechanism.

Results: A total of 95 patients with 107 lesions underwent coronary interventions with NSE. Of these, 72 lesions (12 de-novo lesions and 60 in-stent restenosis) were treated using in-stent dilatation. Two distal LSD cases occurred, representing an incidence of 2.78\% (2/72) among all procedures; $16.7 \%$ (2/12) of the de-novo lesions developed LSD. In vitro experimentation allowed indentification of the mechanisms involved and bailout strategies.

Conclusions: This is the first study to evaluate NSE balloon catheter entrapment complicated by distal LSD in which reconstruction of the deformed stent and retrieval of the NSE could be achieved successfully. There is a potential hazard for distal LSD during post-dilatation using the NSE balloon due to its structural characteristics. Careful assessment is needed to prevent this complication. (Cardiol J 2019; 26, 6: 645-652)
\end{abstract}

Key words: coronary artery disease, balloon angioplasty, complication, drug eluting stent

\section{Introduction}

Recent advances in intracoronary drug-eluting stents (DES) have led to the development of the thin strut platform with fewer connectors. This has improved delivery and conformability during percutaneous coronary intervention (PCI). However, several investigations, including bench testing, have increasingly reported a new complication of longitudinal stent deformation (LSD) because these new stent designs have reduced longitudinal strength [1-4]. LSD is defined as the distortion or shortening of intracoronary stents after successful stent deployment. This can occur in three possible patterns: 1) deformation with intra-stent wrinkling and overlapping of the proximal and distal stent fragments within a single stent; 2) deformation with elongation and 3) deformation with shortening [5]. The fundamental cause of LSD is impact from any secondary device through a stent and

Address for correspondence: Hiroki Shibutani, MD, Division of Cardiology, Osaka Saiseikai Izuo Hospital, 3-4-5 Kitamura, Taisho-ku, Osaka 551-0032, Japan, tel: 81-6-6552-0091, fax: 81-6-6552-0091, e-mail: hs.vxvii@gmail.com 
Table 1. The incidence of distal longitudinal stent deformation caused by non-slip element balloon.

\begin{tabular}{lccc}
\hline & De-novo lesion & In-stent restenosis & Total \\
\hline No. of lesions & 12 & 60 & 72 \\
No. of stent deformations & 2 & 0 & 2 \\
Incidence of stent deformations & $16.70 \%$ & - & $2.78 \%$ \\
\hline
\end{tabular}

contact with a guide catheter in a proximally deployed stent. This is more common in complex lesions such as ostial, bifurcation, left main trunk, and severely calcified lesions, and is also associated with the use of an extra-support guiding catheter and extra guidewires [6, 7]. However, unusual cases of distal LSD were experienced caused by a Lacrosse ${ }^{\circledR}$ non-slip element (NSE) balloon (Goodman Co., Ltd., Nagoya, Japan). The mechanism of this type of LSD is strongly associated with structural features of the NSE balloon itself. The NSE has three flexible nylon elements placed along the outside of the balloon every $120^{\circ}$ for scoring effect while dramatically reducing slippage during inflation for in-stent restenosis, calcified, tapered, tortuous, bifurcated, and ostial lesions. Proximal and distal connections attach the elements and balloon catheter. Consequently, spaces exist between the elements and the balloon body [8]. The distal connection carries the potential risk of entrapment in a stent platform during post-dilatation, leading to distal LSD. Herein, is described local clinical experience with distal LSD caused by an NSE balloon, and discussion of the incidences and mechanisms involved.

\section{Methods}

This is a case series study on cases of coronary intervention cases collected at the documented hospital. The database was retrospectively examined and PCI cases were reviewed using the NSE balloon between May 2014 and June 2017. All PCI procedures were recorded on a digital computerbased program, and data concerning distal LSD caused by the NSE balloon were identified from this digital archive. The definition of distal LSD is the shortening of the distal portions of intracoronary stents after in-stent dilatation using an NSE balloon. Intravascular imaging devices, such as intravascular ultrasound (IVUS) or optical coherence tomography (OCT), were used during all PCI procedures at three common time points: pre-stenting, post-stenting (in the case of in-stent restenosis, only pre-dilatation), and after postdilatation using the NSE. The identification of LSD cases were performed manually by screening these angiographic findings and the IVUS or OCT imaging databases, and the incidence was estimated. In addition, a bench test was performed to identify the mechanism underlying this type of LSD using silicon tube models. As an in vitro assessment, the distal LSD was reproduced and was caused by the NSE balloon.

\section{Results}

During the study period, a total of 95 patients, with 107 lesions, underwent PCI using the NSE balloon for acute coronary syndrome or stable angina. Thirty-five lesions were excluded because the NSE balloon was used only for pre-dilatation; therefore, in-stent dilatation using the NSE balloon was performed for 72 lesions (12 de-novo lesions and 60 in-stent restenosis). When all procedures were considered, distal LSD, caused by the NSE balloon occurred in 2 cases. Table 1 shows that the incidence of stent deformation was $2.78 \%$ (2/72) among all procedures and $16.7 \%(2 / 12)$ when considering only de-novo lesions. The true incidence of this type of LSD remains unknown due to the small study population; however, a high incidence rate was found during procedures for de-novo lesions. On the other hand, this complication did not occur in patients being treated for in-stent restenosis. The 60 cases of in-stent restenosis were detected by performing follow-up coronary angiogram 6 to 20 months after stenting. All the previous stents were sealed by neointimal tissue from OCT findings, therefore, it might be impossible that the NSE was entrapped in the distal edge of the stent. Table 2 summarizes the clinical and procedural details of patients with de-novo lesions. Both LSD cases were not complex, but simple de-novo lesions and stent deformations occurred at the distal edge of the stent when performing post-dilatation with the NSE balloon and pulling it back. The operators detected the stent deformations by fluoroscopy and IVUS or OCT images and could successfully bail-out to perform further dilatation with a noncompliant balloon. There were no adverse events during the procedural clinical course. 


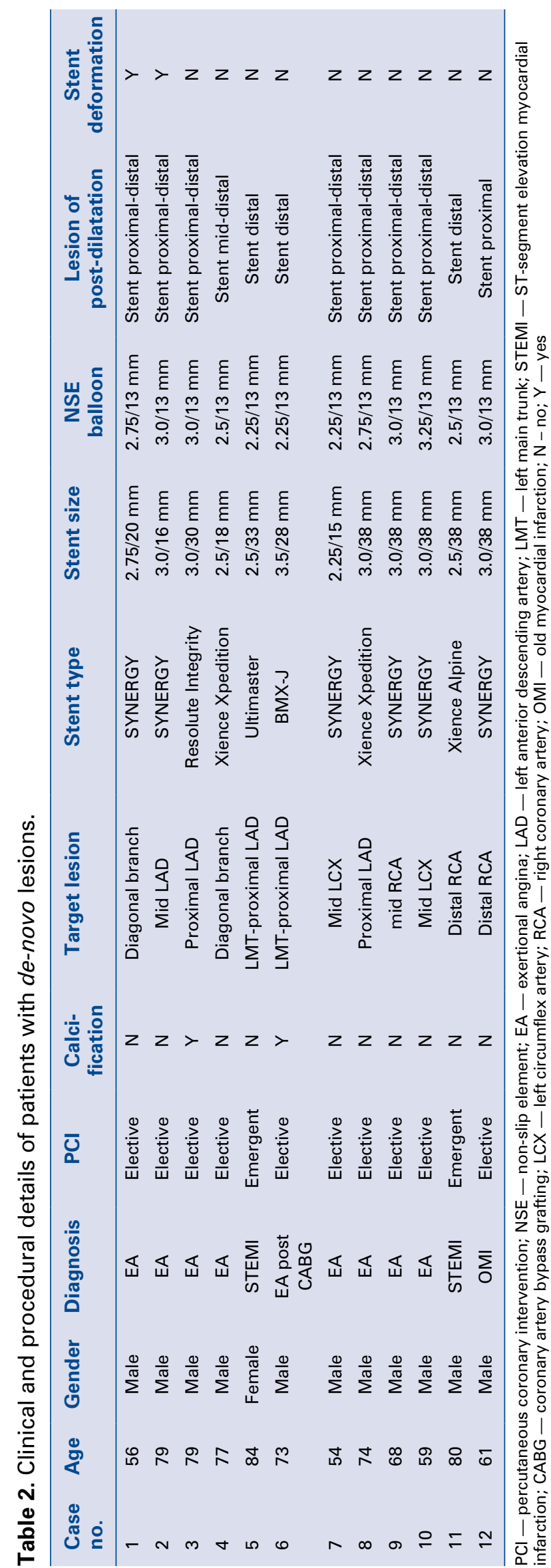

The specific details of the 2 cases are described below.

\section{Case 1}

A 56-year-old man was admitted with chest pain. He was diagnosed with exertional angina based on diagnostic coronary angiography and underwent PCI of the mid-left anterior descending (LAD) artery and the first diagonal branch, respectively (Fig. 1A). The procedure began with the diagonal branch. First, pre-dilatation was attempted using a 2.75/13 mm NSE balloon, but the device could not be introduced through the target lesion due to tortuosity of the diagonal branch. Pre-dilatation was then performed using a $2.75 / 15 \mathrm{~mm}$ non-compliant balloon, and a $2.75 / 20 \mathrm{~mm}$ SYNERGY stent (Boston Scientific, Natick, MA, USA) was deployed at nominal pressure without protrusion to the LAD artery. IVUS revealed distal stent malapposition (Fig. 1B). Post-dilatation was therefore performed with the same NSE balloon. Delivery to the distal end of the stent was performed without complication and postdilatation was completed at rated burst pressure (18 atm). However, LSD was detected at the distal end of the stent by fluoroscopy after removal of the NSE (Fig. 1C). It was considered that while the NSE balloon was pulled back, the three elements and the distal connection of the balloon were caught in the stent platform. This led to stent deformation, with the distal edge of the stent shortened. The deformed stent was recovered by additional dilatation with non-compliant balloon, which successfully dilated the stent (Fig. 1D). Finally, treatment proceeded with the LAD artery, and final angiogram showed good blood flow.

\section{Case 2}

This patient was a 79-year-old male receiving hemodialysis due to end-stage renal disease. $\mathrm{He}$ underwent a diagnostic coronary angiogram for chest pain during hemodialysis, which revealed severe stenosis of mid-LAD artery (Fig. 2A). He was diagnosed with exertional angina and PCI was performed for the mid-LAD artery. After pre-dilatation with a 3.0/13 $\mathrm{mm}$ NSE balloon, a 3.0/16 mm SYNERGY stent was deployed at nominal pressure. OCT images revealed malapposition at the distal end of the stent (Fig. 2B), and post-dilatation was performed with the same NSE balloon. Delivery of the device into the stent and inflation of the balloon at rated burst pressure (18 atm) was performed without complications; however, the NSE could not be withdrawn after deflation. Therefore, it was pushed distal to the stent once and pulled back 


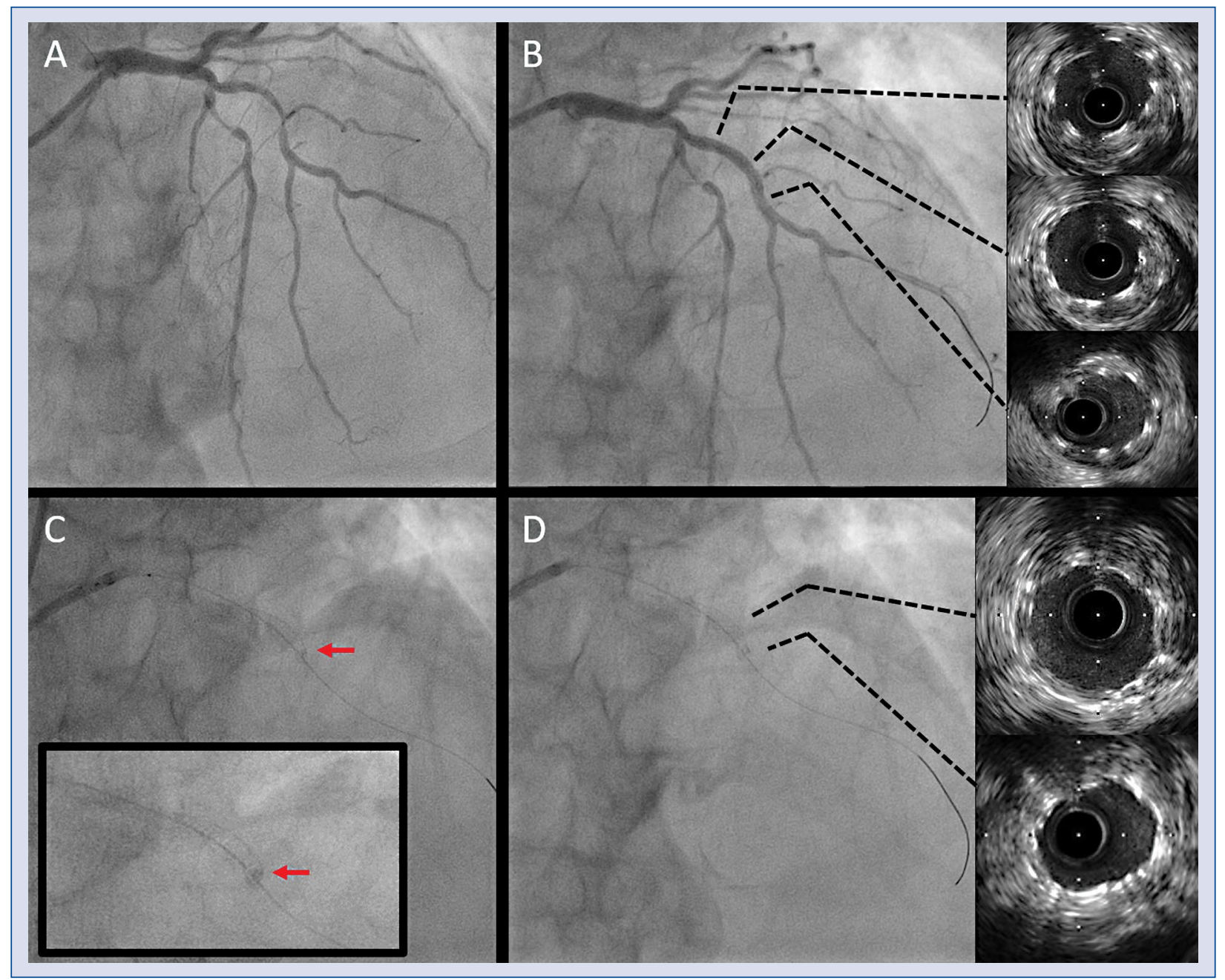

Figure 1. Case 1. A. The pre-procedural coronary angiogram revealed severe stenosis at the left anterior descending (LAD) artery and the first diagonal branch. B. After deployment of a 2.75/20 mm SYNERGY stent (Boston Scientific, Natick, MA, USA) at the diagonal branch without protrusion to the LAD artery, intravascular ultrasound (IVUS) showed distal stent malapposition. C. Post-dilatation using a $2.75 / 13 \mathrm{~mm}$ non-slip element (NSE) balloon was performed. When pulling back the NSE after deflation, slight resistance was encountered. Then longitudinal stent deformation was detected, with shortening of the distal end of the stent, using fluoroscopy after removal of the NSE (red arrow). D. Further dilatation, with a $2.75 / 15 \mathrm{~mm}$ non-compliant balloon, was then performed. Fluoroscopy and IVUS subsequently showed that the deformed stent had been successfully dilated.

again slowly and carefully. After successful removal of the NSE, LSD was identified at the distal end of the stent using magnified fluoroscopy (Fig. 2C) and OCT images (Fig. 2D). Therefore, further dilatation was performed with a $3.25 / 8 \mathrm{~mm}$ non-compliant balloon, which successfully dilated the deformed stent (Fig. 2E, F). After the procedure, the distal connection of the NSE was turned up (Fig. 3). This revealed that the NSE balloon was stuck by entrapment to the distal connection and elements in the stent platform. Finally, the entrapment was successfully relieved by pushing the NSE through the distal stent once.

\section{Bench test report}

Several silicon tube models were used to successfully reproduce distal LSD caused by NSE balloon. Here, 2 typical cases are presented. In the first case, using a bifurcation model (inner diameter 4.0-3.5-3.5 mm), a Runthrough ${ }^{\circledR}$ NS guide wire (Terumo Corporation, Tokyo, Japan) was positioned and a 3.0/28 $\mathrm{mm}$ SYNERGY stent was deployed at nominal pressure (Fig. 4A). Then, we delivered a 3.0/13 mm NSE balloon, which had already been inflated and deflated out of the silicon tube, into the stent. During repeated pushing/pulling, the NSE balloon became entrapped in the distal end of the 


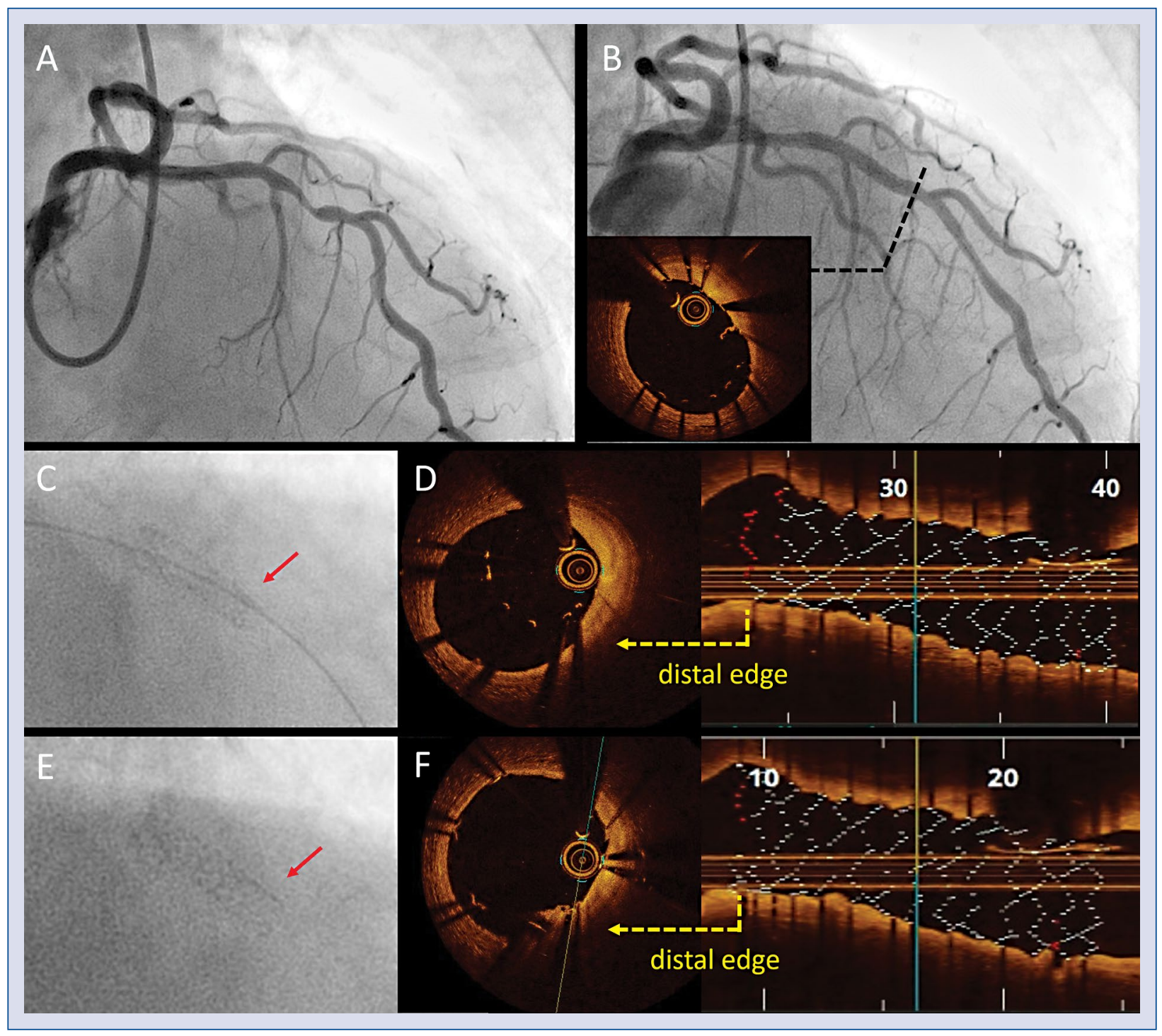

Figure 2. Case 2. A. The coronary angiogram showed severe stenosis the mid-left anterior descending (LAD) artery. B. After deployment of a 3.0/16 mm SYNERGY stent at the LAD artery, optical coherence tomography (OCT) images revealed malapposition of the distal end of the stent. C. After post-dilatation with a 3.0/13 mm non-slip element balloon, longitudinal stent deformation was identified at the distal end of the stent using magnified fluoroscopy (red arrow). D. OCT images revealed shortening of the distal edge of the stent. E, F. After further dilatation with a 3.25/8 $\mathrm{mm}$ non-compliant balloon, the stent was checked for successful reconstruction using magnified fluoroscopy (red arrow) and OCT images.

stent (Fig. 4B). As the NSE was pulled back, the distal stent was gradually shortened (Fig. 4C, D). There was slight resistance; therefore, it was pushed distal to the stent once and pulled back again carefully. The entrapped NSE was successfully removed and distal LSD occurred (Fig. 4E).

In the second case, using a bifurcation silicon tube (inner diameter 3.5-3.5-2.5 mm), a $2.5 / 28 \mathrm{~mm}$ SYNERGY stent was deployed at nominal pressure (Fig. 5A). Delivery of a 2.5/13 mm NSE balloon through the stent was performed without complication (Fig. 5B). However, while pulling back the NSE, it became entrapped at the distal edge of the stent and LSD occurred (Fig. 5C). Although it was pushed to the distal stent once and pulled back again, as in the first case, the NSE was entrapped each time and could not be removed. Therefore, there was an attempt to inflate the NSE balloon at high pressure at the distal part of the stent, and the deformed stent was dilated (Fig. 5D). Accordingly, the NSE was successfully withdrawn through the stent (Fig. 5E). 


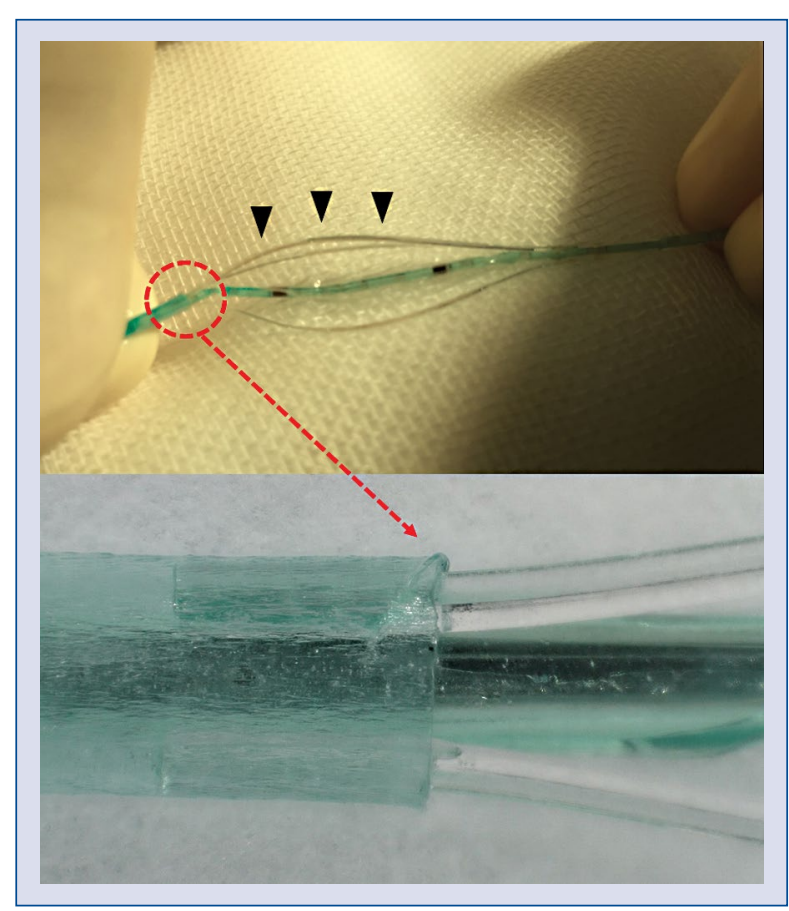

Figure 3. Image of non-slip element (NSE) balloon. After the procedure, the NSE balloon catheter was checked, revealing that the distal connection of the NSE was turned up (red arrow). The black arrowhead shows the structural feature of three elements that are attached only proximally and distally and not bonded to the balloon body.

\section{Discussion}

According to available research this is the first report to describe NSE balloon catheter entrapment complicated by distal LSD in which reconstruction of the deformed stent and retrieval of the NSE could be achieved successfully. There was discussion about the high incidence rate and mechanisms associated with this complication using a combination of database screening and bench test analyses.

Initially, the most important factor related to distal LSD was the structural features of NSE balloon, which becomes easily entrapped in the stent platform. The NSE balloon is beneficial for pre-dilatation in de novo lesions or in stent restenosis lesions because it characteristically features three nylon elements. The elements on the balloon are separated from each other by $120^{\circ}$. These are attached proximally and distally; therefore, the balloon body and elements are not bonded. Although it is plausible that any secondary devices through the deployed stent could cause LSD [1-3], these structural features are assumed to lead to easier entrapment at the stent platform compared with other devices.

Secondly, traction between devices and the distal edge of the stent may induce distal stent shortening. Bench tests indicated that wire bias and an under-expanded distal edge may be associated with LSD in the post-dilatation intravascular scenario. The mechanism by which the NSE caused stent crushing was by wire bias leading to contact between the distal connection and the stent edge. In Figure 4, the distal connection was entrapped at the distal edge of the stent due to wire bias, causing contact between the distal connection and stent edge along the inner curve of the bend. In Figure 5, wire bias caused contact along the outer curve of the bend. At this point, the stent edge may not have been fully embedded in the vessel wall. Thus, wire bias and an under-expanded distal edge might be the primary triggers of NSE entrapment. In such intravascular situations, using the NSE balloon post-dilatation carries a potentially high risk for distal LSD.

On the other hand, the main reason that NSE balloon was used for post-dilatation was only to reduce the total number of balloon devices. Sufficient expansion could achieved by NSE at high pressure when stent and NSE balloon sizes were similar. Inflation at rated burst pressure (18 atm) can make the balloon diameter increase to about a quarter size up $(0.25 \mathrm{~mm})$. However, post-dilatation with NSE at rated burst pressure could produce strong traction between devices which may be associated with the occurrence of LSD. In addition, the position of post-dilatation can have a strong impact on results. According to the mechanism demonstrated in the bench test, LSD can only occur when post-dilation was performed at the distal part of the stents. In clinical practice, NSE can be safely used for post-dilatation by not advancing it to the distal stents.

Other strategies were also considered to overcome entrapment and to aid in NSE balloon removal. The importance of treating LSD has been demonstrated by previous reports in which LSD was thought to be related to a subsequent adverse event, such as stent thrombosis [2, 6]. A careful assessment is also required to analyze the difficulty in removing the NSE balloon from highly deformed stents. If this occurs, some measures are required to extract the trapped device. First, the balloon catheter must not be pulled the by force when resistance is encountered. Pulling with greater force, will result in more deformation of the stent, and the stent will ultimately be crushed. Therefore, push- 


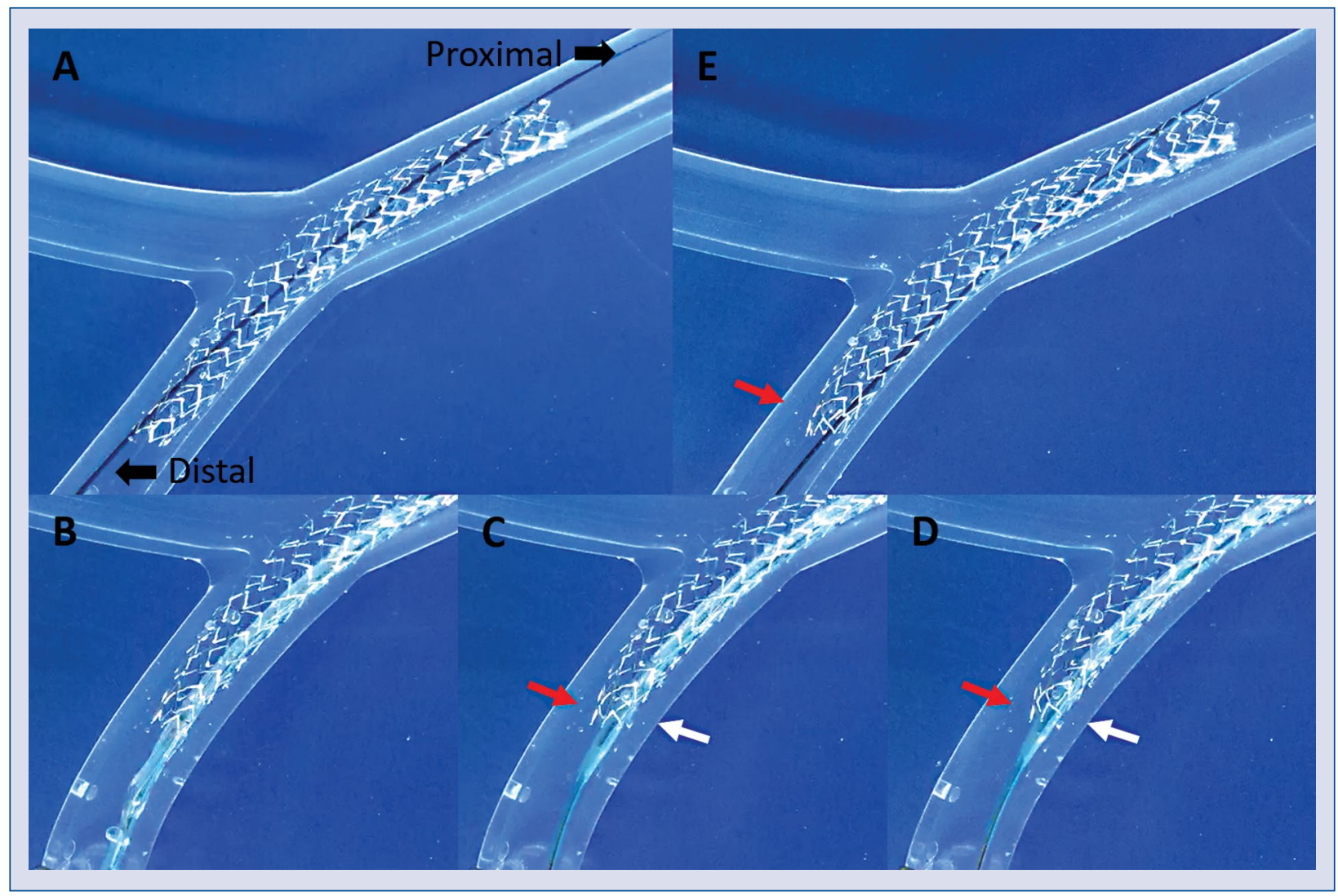

Figure 4. Longitudinal stent deformation (LSD) in case 1 was reproduced in the bench test. A. Using a bifurcation model (inner diameter 4.0-3.5-3.5 mm), the Runthrough ${ }^{\circledR}$ NS guide wire (Terumo Corporation, Tokyo, Japan) was positioned and a 3.0/28 mm SYNERGY stent was deployed at nominal pressure. B. A 3.0/13 mm non-slip element (NSE) balloon was delivered, which had already been inflated and deflated out of the silicon tube, into the stent. C, D. When the NSE was pulled back, the distal connection was entrapped along the inner curve of the bend (white arrow) and the distal stent was gradually shortened (red arrow). E. The catheter was pushed into the distal stent once and pulled back carefully. Then, the entrapped NSE was successfully removed and distal LSD occurred (red arrow).

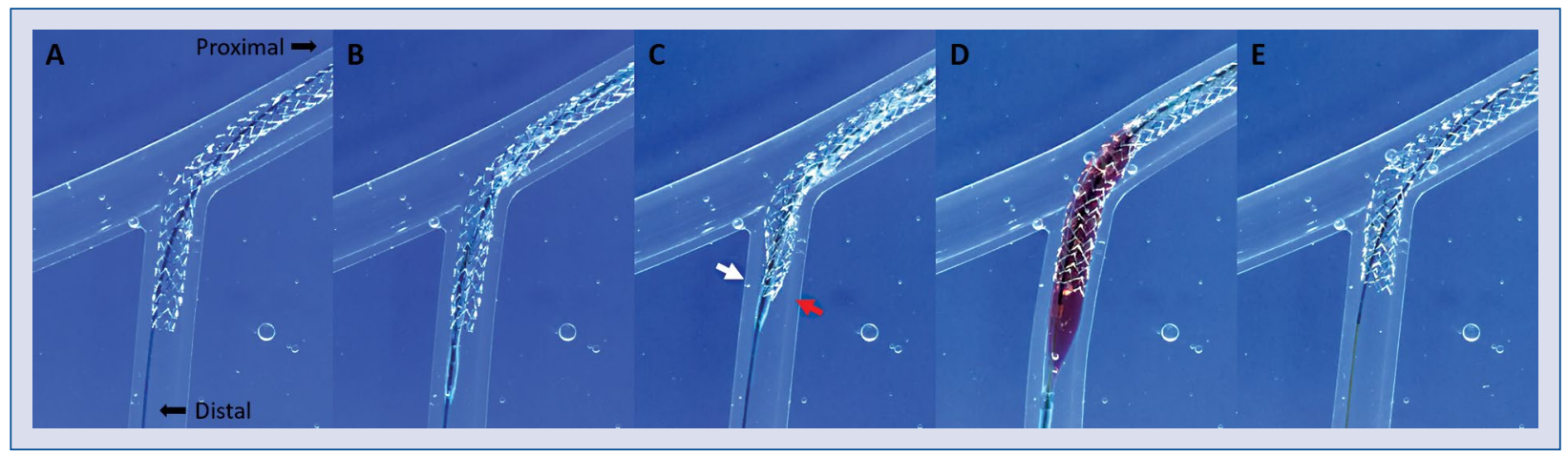

Figure 5. Longitudinal stent deformation (LSD) in case 2 reproduced in the bench test. $\mathbf{A}$. Using a bifurcation silicon tube (inner diameter 3.5-3.5-2.5 mm), a 2.5/28 mm SYNERGY stent was deployed at nominal pressure. B. Delivery of a 2.5/13 mm non-slip element (NSE) balloon through the stent was performed without complications. C. While pulling back the NSE, the distal connection was entrapped in the distal edge of the stent along the outer curve of the bend (white arrow) and distal LSD occurred (red arrow). D. The device could not be removed because the deformation had progressed significantly. Therefore, reconstruction of the deformed stent by balloon dilatation at high pressure was performed. E. Accordingly, the NSE was successfully withdrawn through the stent. 
ing the device distal to the stent once was needed, if possible. Advancing the balloon, may disengage the balloon and stent, and then gentle traction of the entrapped device is a simple and frequently effective technique. Case 2 and Figure 4 show the successful removal of NSE using this method. Second, in cases of severe stent deformation, the device was repeatedly trapped at the stent distal edge every time. Consequently, stent deformation may have progressed significantly. Thus, reconstruction of the deformed stent by balloon dilatation is the primary procedure with which to allow for the safe retrieval of the entrapped device. The NSE will need to be inflated to dilate the deformed stent, which will then permit further attempts at withdrawal. Figure 5 shows successful removal using this method. In the worst case, in which it is impossible to remove the NSE with any method, the patient may require cardiac surgery to extract the trapped device. The clinical outcomes of patients after successful removal of entrapped devices and the reconstruction of deformed stents are relatively good [2].

\section{Limitations of the study}

There are some limitations associated with the present study. Firstly, this was a retrospective single-center study that included a small patient population. Thus, selection bias may exist and could have influenced the conclusion. The results need to be confirmed by a larger multicenter study. Secondly, factors concerning different types of DES were not evaluated in the present study. Several bench testing reports have suggested that longitudinal stent compression is associated with stent design; on the other hand, lower resistance does not always correlate with significantly higher crush rates under clinical conditions [9-13]. In the present study, only the SYNERGY stent was used in the bench test. It remains unclear as to whether differences in stent types are related to the mechanism of LSD. Finally, although successfully treated LSD is not usually associated with adverse events at least in the short-term, the long-term clinical outcome remains unknown.

\section{Conclusions}

In conclusion, there is a significant potential hazard of distal LSD during post-dilatation when using the NSE balloon due to its characteristic structural features. Considering the aforementioned mechanism and bailout strategies, careful clinical assessment is required to prevent entrapment in the stent platform.

\section{Acknowledgements}

We would like to thank the staff of Saiseikai Izuo Hospital. Furthermore, we are indebted to Editage, an editing company, for critical reading of this manuscript.

\section{Conflict of interest: None declared}

\section{References}

1. Hanratty CG, Walsh SJ. Longitudinal compression: a "new" complication with modern coronary stent platforms: time to think beyond deliverability? EuroIntervention. 2011; 7(7): 872-877, doi: 10.4244/EIJV7I7A135, indexed in Pubmed: 21970984.

2. Mamas MA, Williams PD. Longitudinal stent deformation: insights on mechanisms, treatments and outcomes from the Food and Drug Administration Manufacturer and User Facility Device Experience database. EuroIntervention. 2012; 8(2): 196-204, doi: 10.4244/EIJV8I2A33, indexed in Pubmed: 22381263.

3. Williams PD, Mamas MA, Morgan KP, et al. Longitudinal stent deformation: a retrospective analysis of frequency and mechanisms. EuroIntervention. 2012; 8(2): 267-274, doi: 10.4244/EIJV8I2A41, indexed in Pubmed: 22052084.

4. Janakiraman E, Subban V, Victor SM, et al. Longitudinal deformation - price we pay for better deliverability of coronary stent platforms. Indian Heart J. 2012; 64(5): 518-520, doi: 10.1016/j. ihj.2012.07.012, indexed in Pubmed: 23102394.

5. Inaba S, Weisz G, Kobayashi N, et al. Prevalence and anatomical features of acute longitudinal stent deformation: An intravascular ultrasound study. Catheter Cardiovasc Interv. 2014; 84(3): 388-396, doi: 10.1002/ccd.25411, indexed in Pubmed: 24478182.

6. Guler A, Guler Y, Acar E, et al. Clinical, angiographic and procedural characteristics of longitudinal stent deformation. Int J Cardiovasc Imaging. 2016; 32(8): 1163-1170, doi: 10.1007/s10554016-0905-1, indexed in Pubmed: 27198891.

7. Arnous S, Shakhshir N, Wiper A, et al. Incidence and mechanisms of longitudinal stent deformation associated with Biomatrix, Resolute, Element, and Xience stents: Angiographic and case-by-case review of 1,800 PCIs. Catheter Cardiovasc Interv. 2015; 86(6): 1002-1011, doi: 10.1002/ccd.25790, indexed in Pubmed: 25533972.

8. Taguchi I, Kageyama M, Kanaya T, et al. Clinical significance of non-slip element balloon angioplasty for patients of coronary artery disease: a preliminary report. J Cardiol. 2014; 63(1): 19-23, doi: 10.1016/j.jjcc.2013.06.009, indexed in Pubmed: 23906528.

9. Prabhu S, Schikorr T, Mahmoud T, et al. Engineering assessment of the longitudinal compression behaviour of contemporary coronary stents. EuroIntervention. 2012; 8(2): 275-281, doi: 10.4244/ /EIJV8I2A42, indexed in Pubmed: 22057097.

10. Ormiston JA, Webber B, Webster MWI. Stent longitudinal integrity bench insights into a clinical problem. JACC Cardiovasc Interv. 2011; 4(12): 1310-1317, doi: 10.1016/j.jcin.2011.11.002, indexed in Pubmed: 22136972.

11. Ormiston JA, Webber B, Ubod B, et al. Stent longitudinal strength assessed using point compression: insights from a second-generation, clinically related bench test. Circ Cardiovasc Interv. 2014; 7(1): 62-69, doi: 10.1161/CIRCINTERVENTIONS.113.000621, indexed in Pubmed: 24368821.

12. Barragan P, Garitey V, Mouneimne K, et al. Longitudinal compression behaviour of coronary stents: a bench-top comparative study. EuroIntervention. 2014; 9(12): 1454-1462, doi: 10.4244/ /EIJV9I12A243, indexed in Pubmed: 24755385.

13. Abdel-Wahab M, Sulimov DS, Kassner G, et al. Longitudinal deformation of contemporary coronary stents: an integrated analysis of clinical experience and observations from the bench. J Interv Cardiol. 2012; 25(6): 576-585, doi: 10.1111/j.15408183.2012.00765.x, indexed in Pubmed: 23017115. 